\title{
Moral Obligations in Head and Neck Allo-Transplantation Innovation
}

\author{
Carmen Paradis
}

Published online: 7 June 2014

(C) Springer Science+Business Media New York 2014

\begin{abstract}
When a major surgical innovation such as head and neck allo-transplantation has the potential for generalizable applicability, the ethics of research govern its development, use, assessment, and early promulgation. Research ethics requires that the science of the research be rigorous in terms of conduct and assessment in order to created reliable unbiased evidence. However, because such research requires human subjects, ethical constraints must be in place for their protection. In head and neck allotransplantation, this requires an orientation that differs from clinical practice as well as from what is found in research on established procedures. This different orientation impacts more than just the surgical team. It also involves those responsible for subject assessment and protection, study intervention parameters, overview, and data analysis. This paper discusses why such an altered approach is required, how to operationalize it, and some of the difficulties in doing so.
\end{abstract}

Keywords Surgical research - Head and neck allo-transplantation · Surgical innovation .

Research ethics · Surgical ethics

\section{Introduction}

Surgeons, frustrated by their inability to re-create structures such as an amputated tongue, a larynx, or a face destroyed by trauma or malignancy, have turned to allo-transplantation as an innovative solution. Such well-meant intentions,

C. Paradis $(\bowtie)$

Department of Bioethics, Cleveland Clinic, 9500 Euclid Ave, Cleveland, OH 44195, USA

e-mail: paradic@ccf.org even when supported by good reasoning and preliminary planning and performed by skilled surgeons, are not sufficient alone to justify individual surgeons proceeding with these procedures. More is required. This article will explain what is required and why.

\section{Surgical Innovation}

Innovation is integral to surgical practice. Surgeons think of a better way to do something, then do it. In the surgical culture, such innovation is seen as a professional responsibility to maximize benefit and reduce risk for a patient. This surgical problem solving is part of the attraction and satisfaction of being a surgeon. When such innovation is minor or done to treat a unique patient in a particular situation, the ethics of clinical care govern its use. However, when an innovation has the potential to be applied to a broader population, that is, has more generalizable applicability, the ethics of research govern its development, use, assessment, and early promulgation. Such is the case with allo-transplantation in the head and neck.

\section{Researchers' Moral Obligations}

Research, especially in its early innovative stages, involves more risk and less personal benefit than standard care, with more uncertainty about both. Ethical justification for such endeavors requires appropriate safeguards. Miller and Joffe $[1 \bullet \cdot]$ have outlined a process to examine the moral obligations of human subjects research which is also applicable to surgical innovation. Their framework is based on the premise that while the science in human subjects research must be rigorous in terms of conduct and assessment in 
order to produce generalizable knowledge, ethical constraints are required because of the involvement of human subjects, who are also often sick patients. Those ethical constraints consistent with the basic ethical principles of clinical practice-beneficence, non-maleficence, respect for persons, and justice-are modified by a scientific orientation rather than a therapeutic one.

Beneficence in the research setting necessitates that the question to be answered by the research be applicable to a population rather than just to an individual. It is of paramount importance that the science of research be rigorous and its reporting honest and accurate if it is to fulfill this obligation to benefit society. Research need not benefit an individual subject, but if it does not, its risks must be justified by the value of the knowledge to be created either for the population as a whole or for the population which is effected by the subject's condition. This is the case in phase I pharmaceutical trials and in research involving normal subjects. The ethical justification of balancing risk to the individual with benefit to society permeates the moral assessment of research. In head and neck allo-transplantation, it means that surgeon-researchers must have the expertise to conduct research that will answer the research question so as to benefit society while maximizing patient-subject benefit to the extent possible within a justifiable research design.

Non-maleficence in research does not mean that research cannot entail harms or burdens but rather that the risk of harm and the additional burdens should be minimized by research design and justified by the value of the knowledge to be created. At this early stage of head and neck allo-transplantation, it is inevitable that patient-subjects will be at greater risk of complications, and that some of those complications may be severe [2]. Burdens, such as increased testing for the sake of knowledge, must be justified by the value of that knowledge. These increased burdens and risks of harm to patient-subjects must be recognized by those who take on this research and by those who oversee it. Attempts must be made to mitigate them.

Respect for persons necessitates that researchers truthfully explain the research goal, its potential benefits, risks, and burdens to subjects, and that they assess the subject's understanding in relation to his/her goals and motivations. To do that researchers must first be honest with themselves about the increased risks and decreased benefits of innovation. It is essential that therapeutic misconception, subjects' belief that the investigators conducting clinical research are providing routine medical care, be addressed and dispelled to the extent possible. Patient-subjects, especially in this innovative stage, need to understand the difference between research and clinical care goals and the uncertainty regarding personal benefit and risk in research, in order to give informed consent. This is especially difficult in head and neck allo-transplantation, where potential research subjects are patients desperately seeking a solution for a difficult problem and whom, as physicians, we feel a professional obligation to help.

Justice requires a "fair" distribution of benefits and burdens in society. This orientation differs from the individualized patient care surgeons are accustomed to practicing because it concerns society as a whole. Injustice has sullied research endeavors in the past in two respects. In some research projects, such as Willowbrook [3], Tuskegee [4], and during the cold war radiation experiments [5], vulnerable people, those unable to protect their own interests, have been subjected to research risks with no or minimal prospect of benefit and without adequately informed, voluntary consent. In these situations, people who were desperate or powerless were subject to the risks of research, sometimes without being informed, sometimes by being misinformed, and sometimes because they felt unable to question those to whom they had turned for help. In these instances, vulnerable people were exploited for the benefit of society-an injustice. On the other hand, there is concern that denying research participation to vulnerable sub-populations leaves such populations without the benefit of the knowledge that societal resources create. This is seen in the pediatric population, where we have failed to involve children in research and as a result have garnered insufficient knowledge about dosing, benefit, and risk of many medications that they need for treatment. To balance the need for vulnerable people to benefit from the knowledge research creates with the exhortation to not exploit them, such vulnerable people should be the subjects of early stage or innovative research only if it addresses a condition that is unique to them. Otherwise their involvement in research should be limited to a time when the safety parameters have been delineated by early stage research done with the less vulnerable.

\section{Applications of Moral Obligations to Head and Neck Allo-Transplantation}

Head and neck allo-transplantation at its current stage of development has features which do not fit neatly into the standard research paradigm. A surgeon is necessarily the instrument of these interventions. This creates an intimacy between a surgeon and surgery which does not exist with a pharmaceutical agent. This is exacerbated in surgical innovation where a surgeon is, in essence, both the creator and vehicle of the research intervention. Inevitably this can create bias in research design, assessment, interpretation, and reporting of results which has the potential to undermine knowledge quality and impact subjects' welfare. Some consider this as an inevitable conflict of interest which requires management [6]. 
Multi-disciplinary involvement in these processes, especially if those other disciplines are autonomous from the surgical team, can help manage this.

\section{Research Design}

Because head and neck allo-transplantation is evolving, its research design requires greater flexibility than what is seen in the systematic investigation in which the Department of Health and Human Services (DHHS) uses to define research [7]. A surgeon who embarks on such innovation must be committed to learning, not from the analysis of a group of outcomes as happens in traditional research, but rather from each case and then appropriately modifying subsequent cases so as to maximize benefit and minimize harm. To do this, s/he must create an accurate, unbiased, and secure record of the pre-intervention state, of the intervention itself, and of the outcomes. This can be very challenging. Some measurements, such as those of appearance in face transplant, are be necessity, subjective, rather than objective. Blinding is in most cases not possible. These factors can lead to biased interpretations of outcomes. Variability in procedure descriptions, inconstant measurements of pre- and post-intervention states, and incompleteness of reporting, and the inevitable influence of different learning curves in the non-standardized hands of multiple surgeons and surgical teams can result in inaccurate or deficient reporting. The ongoing procedural modifications which are inevitable and necessary in such innovative work, can lead to inefficiencies within the system, which make it difficult to adequately inform subjects, minimize harm to them, and maximize benefit to them and to society. One approach for mitigating these problems is to prospectively develop a registry, which contains as many objective parameters as possible, includes source document verification, and has the participation of all involved surgeons [8•, 9-11]. This is an approach in which surgeons are increasingly utilizing in order to better understand the outcomes of established surgical interventions such as orthopedic total joints, urogenital mesh, and many cardiovascular devices [6, 12-14].

It should also be used for innovative surgical research.

\section{Research Subjects}

The benefits, risks, and burdens of head and neck allotransplantation at this early stage are conjectural. How to enhance those benefits and minimize risks will eventually be delineated but at this time is unknown. This requires that only patients who are not reasonable candidates for reconstruction by conventional methods should be considered as potential subjects for this innovation, as the standard alternatives have a more reliable risk-benefit profile $[15,16]$. This does not mean that patients should have necessarily failed previous reconstruction attempts but rather that conventional reconstruction is not feasible or would be unlikely to achieve a sufficiently satisfactory goal. That factor decided, other patient-subject characteristics require evaluation. Given these procedures' complexity and uncertainty regarding benefits and risks, head and head and neck allo-transplantation subjects should have the capacity to understand what is known about this innovation and appreciate the large degree of uncertainty about its outcome. Evidence suggests that those who have shown an ability to develop good coping skills are more likely to adapt to future changes, including the uncertainties of innovations such as facial reconstruction [15-18]. Thus, to maximize benefit and minimize harm, potential subjects' require good coping skills. As with any medical intervention, a concordance of a patient's goals with realistically achievable goals is essential to maximize benefit [16]. It is important to remember that benefit in this respect is as the patient, not the research team, perceives it. To reduce allo-transplantation's risks, especially those of immunosuppression, subjects will need to be compliant with the post-transplant medical regimen and vigilant about immunosuppression's sequelae. Although such compliance cannot be reliably determined pre-operatively, certain factors in solid organ transplantation have been shown to be significant in predicting post-transplant non-compliance. These are adolescence, pre-operative non-compliance, absence of a good social support system, and substance abuse [15-17]. This is not to say that those without these characteristics, many of whom are disadvantaged in other ways in our society, will never be candidates for these interventions but rather that at what is essentially a proofof-concept stage, the risks to those with these characteristics unjustifiably outweigh the benefits. Those benefits include those to society, as an increased likelihood of noncompliance would introduce an unnecessary variable that decreases that benefit by jeopardizing the integrity of the knowledge to be produced, the overarching goal of research innovation. Ultimately, with the development of more reliable knowledge about head and neck allo-transplantation, such individuals will become candidates for such an intervention, even as a research intervention, especially if one of the goals of such research is especially applicable to them such as enhancing compliance in their population.

\section{The Head and Neck Allo-Transplantation Team}

The head and neck allo-transplantation team has the responsibility to produce generalizable knowledge for society, while minimizing harms and enhancing benefits for well-informed patient-subjects who understand and 
accept the risks and goals of the research. These moral obligations intermingle clinical and research skills and orientations. This is one source of patient-subject therapeutic misconception. This confluence of clinical assessment with research goals can minimize patient harms, but it also has the potential to exacerbate them by biasing clinician-researchers' perception of the significance of clinical findings. Ideally, to minimize therapeutic misconception, different professionals would be involved in treating patients and in evaluating them as research subjects; however, in many cases that is not feasible.

Planning and executing these research interventions requires a skillful surgical team, transplantation experts, and relevant rehabilitation experts such as speech pathologists. These roles entail both clinical and research responsibilities. Although experts who assess pre- and postintervention results, such as neurologists or speech pathologists, have primarily a research role in that they acquire research data and identify issues, they may also use their clinical orientation to make recommendations for further interventions to minimize harms or burdens or enhance benefits. Other professionals, such as social workers and psychologists, first assess a potential subject's social and psychological suitability for such an innovation then continue in this research role in the objective assessment of the social and psychological parameters of subjects before and after the intervention. However, they also use a therapeutic orientation to assess and provide any necessary post-intervention support to minimize harms.

Although each group of clinician researchers must be somewhat independent to minimize bias, the groups also must, in most cases, work together both to benefit the patient and create knowledge. For instance, the professionals who assess subjects' psychological state must understand from the surgeons, medical clinicians, and rehabilitation specialists what the recovery process will entail and devise ways to minimize psychological harms.

The areas where some separation between clinical care and research activities can be achieved are in the evaluation of the informed consent process and development of the research registry.

Unlike many other types of research, in an innovation such as head and neck allo-transplantation, which involves vulnerable patient-subjects, it is important to have a person who can assess the adequacy of these patient-subject's understanding of what is involved in and may be achieved by this innovation. Although this person must have contact with the intervention team in order to understand what is involved for the patient-subject, s/he should be uninvolved in the clinical care and evaluations of the patient. This provision is consistent with what is often required in other research involving vulnerable people [14]. Although candidates for head and neck allo- transplantation are not traditionally considered to be vulnerable, their desperation may make them especially susceptible to therapeutic misconception. That perception is heightened by the intermingling of clinical and research functions done by the same individuals in the same clinical setting and by the intimate relationship in the surgical team, in particular, has with the development and execution of the research intervention. Ethically, robust informed consent in head and neck allo-transplantation is a complex process which involves conveying information, evaluating understanding, and dispelling misperceptions. As part of this, a patient-subject needs to consider if participation is consistent with his/her own values [17, 19]. Especially, in the case of facial allo-transplantation, a patient-subject's thoughts and feelings about having the face of a dead person become part of their own body must be explored and ultimately be acceptable to them. Unlike in most other research, the consequences of a possible loss of confidentiality and privacy must be considered in innovative research. Although attempts can and should be made to protect patient-subject identity, patient-subjects interact with the rest of society, despite the best interests of the medical team it is inevitable that someone will talk about such novel procedures. Not only must a plan be pro-actively formulated to manage this information, but also patient-subjects must be aware of and comfortable with the plan and its consequences [20]. This process of educating and exploring values and understanding must occur over time in order for patient-subjects to be able to give informed consent.

The individuals who design the registry do not need any contact with research subjects. They do need to understand the parameters of what is being evaluated or measured. To create the societal benefit of increased knowledge, a mechanism must be prospectively established to accurately collect and analyze all data surrounding the intervention from initial subject assessment to long-term outcomes, including the specificities of all interventions. Ascertaining what data is relevant will be difficult initially. Accomplishing this requires expertise in registry development and analysis. Efficient production of knowledge about these innovations will minimize harms and enhance benefits for future subjects and patients. Doing that requires that the different teams who may embark on these innovations co-ordinate their data collection and analysis pre-emptively so that they can learn from each other rather than collectively making the same mistakes. All of this requires a personal and institutional commitment to knowledge production despite any other conflicts which may exist.

Accomplishing all of this requires significant resources both to protect patient-subjects and create knowledge. It is important to ascertain that there are sufficient financial resources to do both these things. 


\section{Oversight}

A principle of human subject research is that there be oversight from those not involved in the research to objectively evaluate risk-benefit, to ensure that the informed consent process is adequate and to ascertain that the selection process is just. These research ethics principles are embedded in the Belmont Report [21] and the World Medical Association's Declaration of Helsinki [22]. Typically, in the United States, Institutional Review Boards (IRBs) have this role. In their evaluative process, they may suggest mechanisms to enhance benefit, reduce potential for harm, and enrich the informed consent process. IRBs' are under the auspice of DHHS. DHHS's definition of research is that it be a systematic investigation. IRBs can interpret this to limit their oversight of innovation because such evolving endeavors are not systematic and often initially involve only a single patientsubject [23]. Nevertheless, some IRBs have reviewed head and neck allo-transplantation [20]. However, with their focus on systematic investigations, especially randomized controlled trials (RCTs), and lack of expertise in evaluating innovation, IRBs may not have sufficient expertise to understand innovative surgical research issues [8•, 24]. This is exasperated by the fact that few surgeons serve on IRBs. This limits IRBs' surgical expertise and exacerbates surgeons' trust in and frustration with IRBs' ability to understand what is involved in surgical innovation. This does not mean that such surgical innovation should not have IRB oversight but rather that such oversight should be augmented by other types of review. IRBs whose institutions are at the forefront of various types of surgical innovation are coming to recognize that their criteria for protecting human subjects should be the same for surgical innovation as it is for early stage research. This means that increasingly IRBs are asking that principal investigators who submit surgical innovation protocols such as those involving head and neck allo-transplantation provide or produce evidence that is similar in scope to that required of early phase pharmaceutical research [25-28]. Although the exact requirements for obtaining this information vary, evidence about benefit and risk, including an evidentiarybased hypothesis about how the risks are both minimized and justified by the benefits is usually required.

Chiefs of surgery, local surgical innovation committees, experts in the field, and specialty society sub-committees have been proposed as alternative overview mechanisms $[8 \cdot, 29]$. However, involving only surgeons in such a process is problematic as their conflicts of interest may create bias [8• 30, 31]. Furthermore, such a process has the potential to focus on an innovation's surgical features while ignoring medical alternatives and/or adjuncts and neglecting its ethical aspects.
A reasonable solution would be a small ad-hoc innovation committee assembled to evaluate a given innovation in a manner similar to how Data Safety Monitoring Boards (DSMBs) evaluate systematic research. Some committee members should have head and neck surgery expertise, some expertise in corresponding medical fields such as speech pathology in the case laryngeal transplantation and some ethical expertise. Such a group would have the skills to protect human participants, assess a project's capacity to advance knowledge, and understand the surgical milieu sufficiently to facilitate the process. An excellent example of this is the group that designed the trial to evaluate lung reduction surgery for emphysema [32•].

\section{Conclusion}

Surgical innovation such as head and neck allo-transplantation is a multidimensional relationship which involves surgeon-researchers both treating patients and conducting research with research subjects. Although this is not unique to surgical research, there are features of surgical innovation, which increase the complexity of this situation. The research team, especially the surgical team, is heavily invested in the outcome because of their intimate creation and actualization of these groundbreaking interventions. Not only do they develop the intervention in the lab over months to years but then they literally make the intervention happen in the operating room with their own hands. This is very different from writing an order for an experimental medication as happens in pharmaceutical research or performing an already developed operation as occurs in standard surgical research. These characteristics make it essential that mechanisms which will move knowledge forward while protecting vulnerable patients, be part of the research process.

\section{Compliance with Ethics Guidelines}

Conflict of Interest Carmen Paradis declares no conflict of interest.

Human and Animal Rights and Informed Consent This article does not contain any studies with human or animal subjects performed by any of the authors.

\section{References}

Recently published papers of particular interest have been highlighted as:

- Of importance

-• Of major importance

1. • Joffe S, Miller FG. Bench to bedside: mapping the moral terrain of clinical research. Hastings Cent Rep. 2008;38(2):30-42. This 
article describes a paradigm for research ethics which is especially pertinent to the challenges of research in the 21st century.

2. Siemionow M, Ozturk C. Face transplantation: outcomes, concerns, controversies, and future directions. J Craniofac Surg. 2012;23(1):254-9.

3. Diamond EF. The willowbrook experiments. Linacre Q. 1973;40(2):133-7.

4. Pence GE. Human subjects: the tuskegee syphilis study. In: Classic cases in medical ethics. McGraw-Hill; 2005. P. 270-300.

5. Advisory Committee on Human Radiation Experiments. Research ethics and the medical profession: report of the advisory committee on human radiation experiments. JAMA. 1996;276(5):403-9.

6. Wall LL, Brown D. The perils of commercially driven surgical innovation. Am J Obstet Gynecol. 2010;202(1):30.e1-4.

7. United States Department of Health and Human Services. Code of federal regulations, title 45 public welfare, department of health and human services, part 46 protection of human subjects. 2009.

8. - Biffl WL, Spain DA, Reitsma AM, et al. Responsible development and application of surgical innovations: a position statement of the society of university surgeons. J Am Coll Surg. 2008;206(3):1204-9. This article specifically addresses an approach to the ethics of surgical innovation.

9. Barkun JS, Aronson JK, Feldman LS, et al. Evaluation and stages of surgical innovations. Lancet. 2009;374(9695):1089-96.

10. McCulloch P. Developing appropriate methodology for the study of surgical techniques. J R Soc Med. 2009;102(2):51-5.

11. Strasberg SM, Ludbrook PA. Who oversees innovative practice? is there a structure that meets the monitoring needs of new techniques? J Am Coll Surg. 2003;196(6):938-48.

12. Sedrakyan A, Marinac-Dabic D, Holmes DR. The international registry infrastructure for cardiovascular device evaluation and surveillance. JAMA. 2013;310(3):257-9.

13. Anderson-Smits C. Developing of the pelvic floor disorder registry (PFDR). FDA presentation. Power Point Presentation. 2012.

14. Morreim EH. By any other name: the many iterations of "patient advocate" in clinical research. IRB. 2004;26(6):1-8.

15. Morris P, Bradley A, Doyal L, et al. Face transplantation: a review of the technical, immunological, psychological and clinical issues with recommendations for good practice. Transplantation. 2007;83(2):109-28.

16. Brill SE, Clarke A, Veale DM, Butler PE. Psychological management and body image issues in facial transplantation. Body Image. 2006;3(1):1-15.

17. Clarke A, Butler PEM. Face transplantation: psychological assessment and preparation for surgery. Psychol Health Med. 2004;9(3):315-26.
18. Rumsey N. Psychological aspects of face transplantation: read the small print carefully. Am J Bioeth. 2004;4(3):22-5 discussion W23-31.

19. Renshaw A, Clarke A, Diver AJ, Ashcroft RE, Butler PE. Informed consent for facial transplantation. Transpl Int. 2006;19(11):861-7.

20. Paradis C, Siemionow M, Papay F, et al. Ethical considerations in the first american face transplant. Plast Reconstr Surg. 2010;126(3): 896-901.

21. National Commission for the Protection of Human Subjects of Biomedical and Behavioral Research. The belmont report: ethical principles and guidelines for the protection of human subjects of research. 1979. http://www.ohsr.od.nih.gov/guidelines/belmont. html. Accessed 31 Aug 2011.

22. World Medical Association. World medical association declaration of helsinki: ethical principles for medical research involving human subjects. 2008. http://www.wma.net/en/30publications/ 10policies/b3/index.html. Accessed 6 Jan 2010.

23. Reitsma AM, Moreno JD. Surgical research, an elusive entity. Am J Bioeth. 2003;3(4):49-50.

24. Agich GJ, Siemionow M. Facing the ethical questions in facial transplantation. Am J Bioeth. 2004;4(3):25-7 discussion W23-31.

25. Dixon JB, Logue J, Komesaroff PA. Promises and ethical pitfalls of surgical innovation: the case of bariatric surgery. Obes Surg. 2013;23(10):1698-702.

26. Sundaram V, Vemana G, Ghayani SB. Institutional review board approval and innovation in urology: current practice and safety issues. BJU International. 2013;113:343.

27. Zorron R, Phillips HN, Coelho D, Flach L, Lemos FB, Vassallo RC. Perirectal NOTES access: "down-to-up" total mesorectal excision for rectal cancer. Surg Innov. 2012;19(1):11-9.

28. Knight JL. Ethics: the dark side of surgical innovation. Innovations (Phila). 2012;7(5):307-13.

29. Reitsma AM, Moreno JD. Ethics of innovative surgery: US surgeons' definitions, knowledge, and attitudes. J Am Coll Surg. 2005;200(1):103-10.

30. Morreim H, Mack MJ, Sade RM. Surgical innovation: too risky to remain unregulated? Ann Thorac Surg. 2006;82(6):1957-65.

31. Bernstein M, Bampoe J. Surgical innovation or surgical evolution: an ethical and practical guide to handling novel neurosurgical procedures. J Neurosurg. 2004;100(1):2-7.

32. - National Emphysema Treatment Trial Research Group. A randomized trial comparing lung-volume-reduction surgery with medical therapy for severe emphysema. $N$ Engl J Med. 2003;348(21):2059-73. This article describes what can be considered a gold standard for ethical surgical research. 